\title{
Silicon-on-insulator microring resonator sensor integrated on an optical fiber facet
}

\author{
Cristina Lerma Arce, Student Member, IEEE, Katrien De Vos, Tom Claes, Student Member, IEEE, \\ Katarzyna Komorowska, Dries Van Thourhout, Member, IEEE and Peter Bienstman, Member, IEEE
}

\begin{abstract}
In recent years portable, sensitive and cheap sensors have gained interest in fields such as medical diagnostics, food quality control, environmental monitoring and drug development. Especially optical sensors in silicon-on-insulator have proven to be very promising, because they have a low limit of detection, are very compact and can be made with cheap mass fabrication techniques. While a lot of progress has been made to implement such sensors in small and easy to use cartridges, many applications require a sensor probe that can perform measurements at locations that are hard to reach. We introduce a method to integrate a silicon-on-insulator photonic sensor on the facet of a standard single-mode optical fiber, and apply this method to a well-known ring resonator refractive index sensor without deteriorating its sensitivity. We thus combined the good performance of silicon-on-insulator sensors with the high mobility of optical fibers.
\end{abstract}

Index Terms - Optical fiber probe sensor, ring resonator, Silicon-On-Insulator (SOI).

\section{INTRODUCTION}

$\mathrm{I}$ $\mathrm{N}$ recent years portable, sensitive and cheap sensors have gained interest in fields such as medical diagnostics, food quality control, environmental monitoring and drug development. Especially optical sensors in silicon-on-insulator have proven to be very promising, because they have a low limit of detection, are very compact and can be made with cheap mass fabrication techniques. Microring resonator sensors stand out as prime candidates among other technologies such as Fabry-Perot cavities, microdisks, or photonic crystal cavities, for achieving high performance in a robust manufacturable manner [1]. The resonance wavelengths of this type of sensor shift for a changing refractive index in the evanescent field of the resonant modes, which can be made specific for a certain application by chemically modifying the waveguide surface.

A lot of progress has been made to implement silicon-oninsulator sensors in small and easy to use cartridges. [1-5].

Manuscript received October 18, 2010 (Write the date on which you submitted your paper for review.) This work was supported in part by of the Belgian IAP project photonics@be.

The authors are with the Photonics Research Group at the Department of Information Technology (INTEC), Ghent University-IMEC, B-9000 Gent, Belgium (e-mail: Cristina.Lermaarce@intec.ugent.be)
However, many applications require a sensor probe that is flexible, portable, and can perform measurements at locations that are hard to reach [6-7].

We introduce a method to integrate a silicon-on-insulator

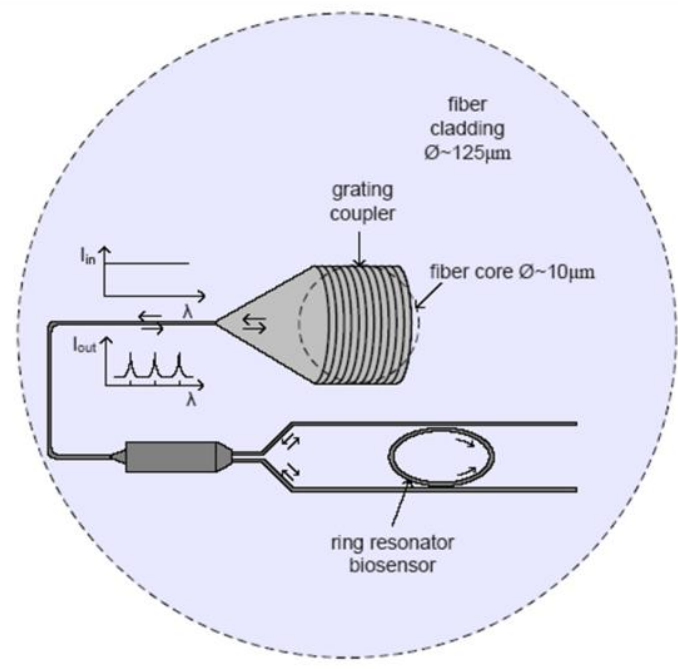

(a)

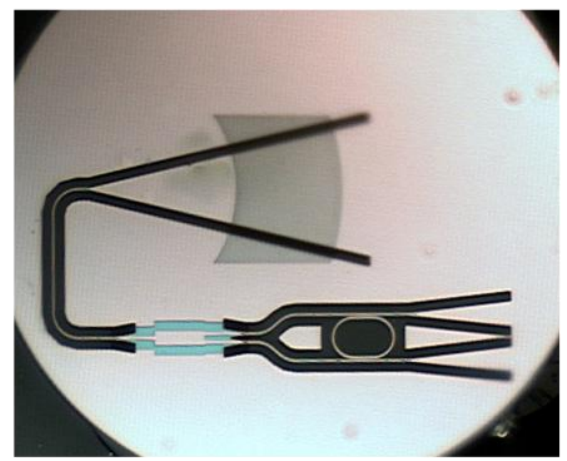

(b)

Fig. 1. (a) Top view of the optical fiber probe: photonic integrated circuit aligned to the core of the optical fiber. The circuit is designed so that light is coupled in and out via the same grating coupler [7] and it is also required to be compact in order to fit on the facet of a standard single-mode optical fiber. A focused grating coupler is used to circumvent the need for a space consuming waveguide taper [8]. A $3 \mathrm{~dB}$ multimode interferometer (MMI) couples light from that grating coupler to both access waveguides of an adddrop ring resonator, which has a compact racetrack shape with $4 \mu \mathrm{m}$ bend radius and $4 \mu \mathrm{m}$ straight sections. At a resonance wavelength, both a clockwise and counterclockwise resonance mode will be excited, causing light to be coupled back in the access waveguides. The same MMI is used to combine the reflected light towards the grating coupler. (b) Microscope picture of the top view of the optical fiber probe. 
photonic sensor on the facet of a standard single-mode optical fiber, and applied this method to a well-known ring resonator refractive index sensor.

\section{DESIGN AND FABRICATION OF THE OPTICAL CIRCUIT}

The photonic chip was fabricated in silicon-on-insulator with $2 \mu \mathrm{m}$ buried oxide and $220 \mathrm{~nm}$ silicon top layer with CMOS-compatible 193nm optical lithography and dry etching [8]. Our device is composed of typical $450 \mathrm{~nm}$ wide photonic wires.

As depicted in Fig. 1(a), we designed the circuit so that light is coupled in and out via a single grating coupler similar to the work presented in [9]. As will be described in section III, we align the fiber core to this grating coupler in a later stage. In order to fit on the facet of a standard single-mode optical fiber, the photonic circuit is also required to be compact. We used a focused grating coupler, which circumvents the need for a space consuming waveguide taper [10]. A $3 \mathrm{~dB}$ multimode interferometer (MMI) couples light from that grating coupler to both access waveguides of an add-drop ring resonator, which has a compact racetrack shape with $4 \mu \mathrm{m}$ bend radius and $4 \mu \mathrm{m}$ straight sections. At a resonance wavelength, both a clockwise and counterclockwise resonance mode will be excited, causing light to be coupled back in the access waveguides. The same MMI is used to combine the reflected light towards the grating coupler.

\section{TRANSFER OF THE PHOTONIC CHIP TO THE FIBER FACET}

In order to transfer the silicon-on-insulator circuit to the facet of a fiber, the sample is first bonded upside down to a silicon carrier wafer by means CrystalBond 509 wax from Electron Microscopy Science. This wax releases easily after processing and leaves only little and easy-to-remove residue on the sensor surface.

In a next step the silicon substrate is removed. First by reducing its thickness to $100 \mu \mathrm{m}$ by mechanical grinding, then to $50 \mu \mathrm{m}$ by dry etching, to finally remove it completely by wet etching. For this wet etching a mixture of acetic, nitric and hydrofluoric acid $\left(\mathrm{CH}_{3} \mathrm{COOH}: \mathrm{NH}_{3}: \mathrm{HF}, 4: 5: 1\right)$ at $20^{\circ} \mathrm{C}$ is used.

At this point only the silicon waveguides and the $2 \mu \mathrm{m}$ thick silicon dioxide layer are left. Then the optical fiber core is actively aligned to the grating coupler through the silicon dioxide layer, after which the chip is glued to the fiber with UV curable resist PAK-01 (Toyo Gosei Co.).

Subsequently, the wax that joined the sensor to the silicon carrier wafer is melted by heating the carrier wafer to $110^{\circ} \mathrm{C}$ with a hot plate. By lifting the fiber, the thin layer of silicon dioxide around the sensor breaks under the weight of the remaining part of the photonic chip, leaving only the sensor circuit on the tip of the fiber. To facilitate breaking the silicon oxide layer around the circuit, we found it necessary to selectively thin down that layer from $2 \mu \mathrm{m}$ to $700 \mathrm{~nm}$ by dry etching prior to the process.

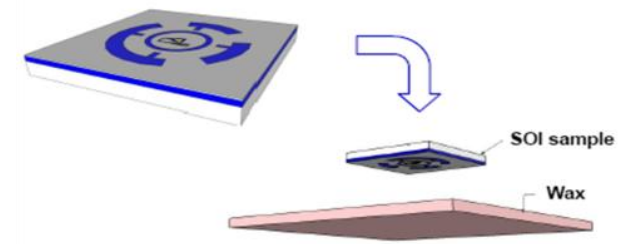

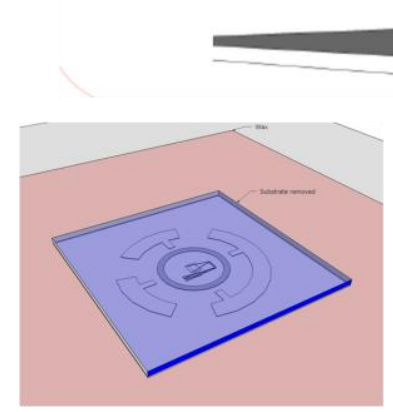

(b) (a)

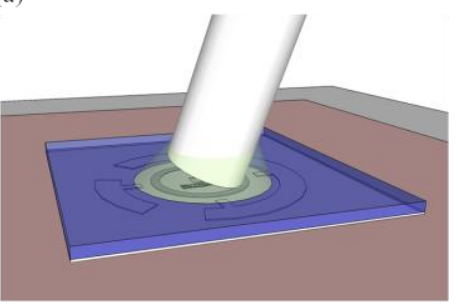

(c)

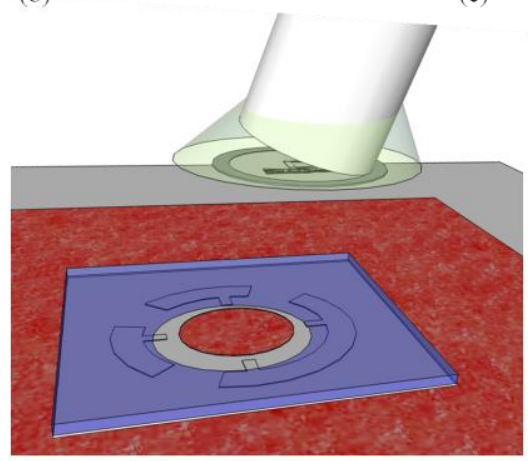

(d)

Fig. 2. Fabrication steps: (a) upside-down bonding of the SOI chip to a silicon carrier wafer by means of wax. (b) removal of the silicon substrate by subsequent mechanical grinding, dry etching and wet etching (c) gluing of the photonic circuit to the tip of a fiber after active alignment of the fiber core to the grating coupler (d) the wax that joined the sensor to the silicon carrier wafer is melted and by lifting the fiber, the thin layer of silicon dioxide around the sensor breaks under the weight of the remaining part of the photonic chip, leaving only the sensor circuit on the tip of the fiber.

Finally, the residues of wax at the sensing surface are removed by immersing the fiber in acetone during $5 \mathrm{~min}$ at 75 ${ }^{\circ} \mathrm{C}$.

\section{CHARACTERIZATION OF THE FIBER PROBE SENSOR}

To measure the transmission of the sensor integrated on the fiber tip, light from a tunable laser source is coupled to our sensor fiber and the light coupled back by the sensor circuit is detected with an optical power meter. The polarization is optimized for maximal coupling to the quasi-TE mode of the SOI circuit using a fiber polarization controller. The bidirectional transmission through the sensor fiber is achieved with a fiber-optic circulator.

Fig. 3 displays a measured resonance peak of the resonator integrated on the fiber tip. By fitting a Lorentzian the quality factor was determined to be 2340, which is lower than that of optimal SOI ring resonators in water. We expect that this can be improved in a future design by optimizing the coupling and by increasing the bend radius of the resonator to reduce the 


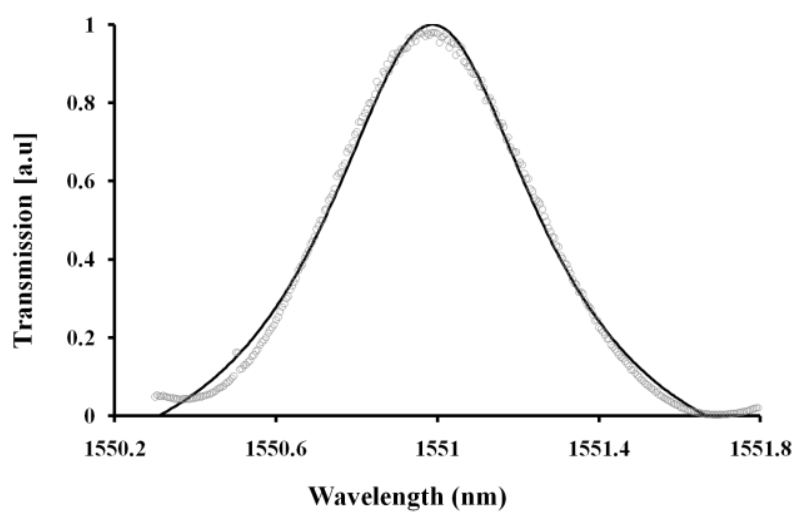

Fig. 3. In grey a measured resonance peak of the resonator integrated on the fiber tip. By fitting a Lorentzian the quality factor was determined to be 2340 , which is lower than that of optimal SOI ring resonators in water. We expect that this can be improved in a future design by optimizing the coupling and by increasing the bend radius of the resonator to reduce the bend loss

bend loss, which can be achieved by making more efficient use of the available space on the fiber tip.

To verify the capability of our fiber probe sensor, we measured its sensitivity for refractive index changes of aqueous solutions and compared it with the one we previously measured for a similar silicon-on-insulator ring resonator sensor that was not integrated on a fiber tip [2]. We used aqueous ethanol solutions with different concentrations, of which we derived the refractive index from [11]. No surface chemistry was applied to the sensor surface.

Fig. 4 illustrates the measured resonance wavelength shift of our sensor as a function of the refractive index change of the solution. A linear fit is depicted with mean wavelength shift values and weighted variance error bars determined over 3 measurements on the same device. The sensitivity is determined to be $70 \mathrm{~nm} / \mathrm{RIU}$, which corresponds with the value we previously measured in [2].

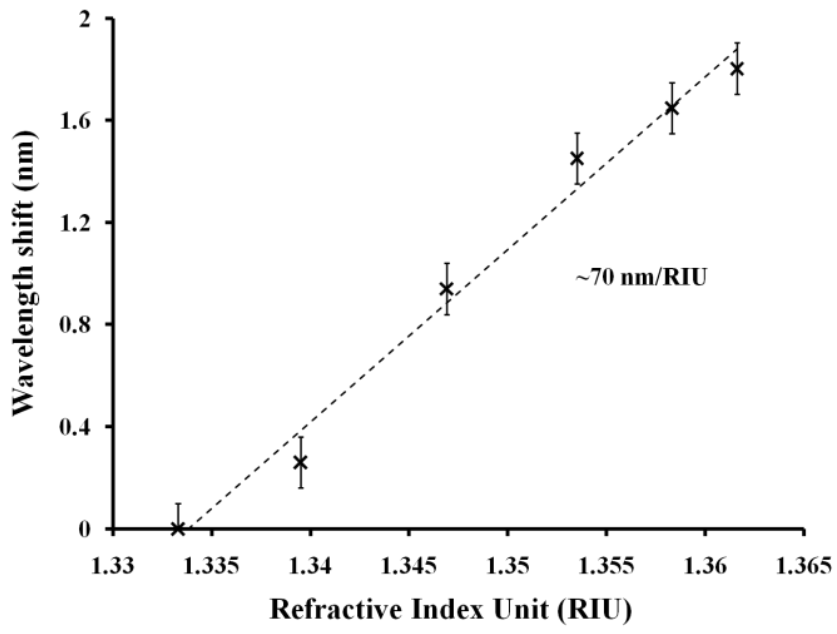

Fig. 4. To verify the capability of our fiber probe sensor, we measured its sensitivity for refractive index changes of aqueous solutions. The mean wavelength shifts over three measurements on the same device are shown with weighted variance error bars. The resonance wavelength shifts linearly with the refractive index of the solution with a sensitivity of $70 \mathrm{~nm} / \mathrm{RIU}$. This value corresponds with values we previously measured on a similar sensor that was not integrated on a fiber facet.

\section{CONCLUSION}

We integrated a well-known silicon-on-insulator ring resonator sensor to the facet of a single mode optical fiber without any degradation of sensitivity, thus combining the good performance of silicon-on-insulator sensors with the high mobility of optical fibers. Future work involves chemical modification of the sensor surface for specific sensing. Among others, a possible application is in-vivo label-free biosensing.

\section{ACKNOWLEDGMENT}

The authors would like to thank ePIXfab (www.epixfab.eu) for the fabrication of the optical chip and S. Verstuyft for fabrication support.

\section{REFERENCES}

[1] M. Iqbal, M.A. Gleeson, B. Spaugh, F. Tybor, W.G. Gunn, M. Hochberg, T. Baehr-Jones, R.C. Bailey, L. C. Gunn. " Label-Free Biosensor Arrays Based on Silicon Ring Resonators and High-Speed Optical Scanning Instrumentation" IEEE Journal of selected topics in quantum electronics, vol. 16(3), pp. 654-661, Mar. 2010.

[2] K. De Vos, I. Bartolozzi, E. Schacht, P. Bienstman, R. Baets, "Siliconon-Insulator microring resonator for sensitive and label-free biosensing",Optics Express, vol. 15(12), pp. 7610-7615, Jun. 2007.

[3] D.X. Xu, M. Vachon, A. Densmore. "Label-free biosensor array based on silicon-on-insulator ring resonators addressed using a WDM approach" Optics letters, vol. 35(16), Aug. 2010.

[4] N.A Yebo, P. Lommens, Z. Hens, R. Baets "An integrated optic ethanol vapor sensor based on a silicon-on-insulator microring resonator coated with a porous $\mathrm{ZnO}$ film" Optics Express, 18(11), p.11859-11866, May 2010

[5] K. De Vos, J. Girones Molera, T. Claes, Y. De Koninck, S. Popelka, E. Schacht, R. Baets, P. Bienstman. "Multiplexed antibody detection with an array of silicon-on-insulator microring resonators" IEEE Photonics Journal, vol. 1(4), pp. 225-235, Oct. 2009.

[6] S. Scheerlinck, D. Taillaert, D. Van Thourhout, R. Baets, Flexible metal grating based optical fiber probe for photonic integrated circuits,Applied Physics Letters, vol. 92(3), pp.031104, Jan 2008

[7] M.Espinosa Bosch, A.J. Ruiz Sánchez, F. Sánchez Rojas, C. Bosch Ojeda "Recent development in optical fiber sensors" Sensors, vol. 7, pp. 797-859, Jun. 2007.

[8] S. K. Selvaraja, P. Jaenen, W. Bogaerts, D. V. Thourhout, P. Dumon, and R. Baets, "Fabrication of photonic wire and crystal circuits in silicon-on-insulator using 193-nm optical lithography," J. Lightwave Technol, vol. 27, pp. 4076-4083, Jan. 2009.

[9] M. Verbist, W. Bogaerts, J. Schrauwen, R. Baets. "Silicon-on-InsulatorBased Retroreflective Optical Marker Chips for Simultaneous Identification and Localization." IEEE Journal of Selected Topics in Quantum Electronics, 15(5), pp.1427-1431, Oct. 2009.

[10] F. Van Laere, T. Claes, J. Schrauwen, S. Scheerlinck, W. Bogaerts, D. Taillaert, L. O'Faolain, D. Van Thourhout, R. Baets, "Compact Focusing Grating Couplers for Silicon-on-Insulator Integrated Circuits" IEEE Photonics Technology Letters, vol. 19(23), pp.1919-1921, Dec. 2007.

[11] R. Belda,.J.V. Herraez, O.Diez. "A study of the refractive index and surface tension synergy of the binary water/ethanol: influence of concentration" Physics and Chemistry of Liquids, vol.43(1), pp. 91-101, Feb. 2005 